\title{
Author Correction: Intrinsic efficiency limits in low-bandgap non-fullerene acceptor organic solar cells
}

Safakath Karuthedath (D), Julien Gorenflot, Yuliar Firdaus (D), Neha Chaturvedi, Catherine S. P. De Castro (D), George T. Harrison, Jafar I. Khan, Anastasia Markina, Ahmed H. Balawi, Top Archie Dela Peña®, Wenlan Liu, Ru-Ze Liang, Anirudh Sharma (D), Sri H. K. Paleti®D, Weimin Zhang, Yuanbao Lin, Erkki Alarousu, Sergei Lopatin, Dalaver H. Anjum (1), Pierre M. Beaujuge, Stefaan De Wolf(D, lain McCulloch (1), Thomas D. Anthopoulos (1), Derya Baran (10, Denis Andrienko (D) and Frédéric Laquai (i)

Correction to: Nature Materials https://doi.org/10.1038/s41563-020-00835-x, published online 23 October 2020.

In the version of this article initially published, Sergei Lopatin (Imaging and Characterization Core Laboratory, King Abdullah University of Science and Technology (KAUST), Thuwal, Kingdom of Saudi Arabia) was omitted from the author list and has now been included for contributing to thin-film imaging and EELS analysis work. In the Fig. 4 caption and fifth from last paragraph of the main text, an error in the second numerator of the IQE equation has been amended to now read: IQE $=\frac{\mathrm{IQE}_{\max }}{2}\left(1+\operatorname{erfc} \frac{\Delta \mathrm{IE}-B}{\sigma}\right)$. Further, in the Supplementary Information file available online, the methods discussed in the Transmission Electron Microscopy section have been updated with an additional figure (Supplementary Fig. 18), with Supp. Fig. 19, 22 citations in the main article updated accordingly.

The changes have been made to the online version of the article.

Published online: 29 November 2021

https://doi.org/10.1038/s41563-021-01178-x

(c) The Author(s), under exclusive licence to Springer Nature Limited 2021 